\title{
Skin tears e a relação com a capacidade funcional em idosos hospitalizados
}

\author{
Relationship of functional capacity and skin tears in hospitalized elderly people
}

Relación de capacidad funcional y skin tears en personas mayores hospitalizadas

Ana Carolina Braga Galvão ${ }^{1}$, Wender Ferreira dos Santos ${ }^{1}$, Andréa Mathes Faustino ${ }^{1 *}$.

\section{RESUMO}

Objetivo: Identificar a ocorrência das lesões de pele do tipo skin tears e verificar sua relação com o estado de funcionalidade em idosos hospitalizados. Métodos: Estudo descritivo realizado em um hospital universitário de Brasília, Distrito Federal, nas unidades de Clínica Médica e Cirúrgica, com idosos internados. Coleta realizada de fevereiro a junho de 2019. Aplicado instrumentos de dados sociodemográficos, avaliação da capacidade funcional, além da realização de exame físico da pele. Resultados: Amostra final de 32 idosos, homens $(56,3 \%)$, média de idade de $68,90( \pm 7,51)$ e pardos $(59,4 \%)$. Na avaliação da capacidade para o autocuidado, $34,5 \%$ eram totalmente dependentes e $18,7 \%$ precisavam de ajuda parcial. Foram observados outros tipos de lesões além das skin tears, considerando que eram idosos acamados e com a pele fragilizada. A prevalência de pacientes com lesão por skin tears foi de $9,4 \%$. Conclusão: A realização do exame físico minucioso na pele em idosos e o levantamento do risco para skin tears durante o período de hospitalização, são ações fundamentais para garantir a segurança no cuidado, além da inclusão da avaliação da capacidade funcional, já que o grau de dependência pode ser um fator de risco para o desenvolvimento deste tipo de lesão.

Palavras-chave: Ferimentos e lesões, Idosos, Atividades cotidianas, Cuidados de enfermagem.

\begin{abstract}
Objective: To identify the occurrence of skin tear skin lesions and to verify their relationship with the functional status of hospitalized elderly. Methods: Descriptive study conducted at a university hospital in Brasília, Distrito Federal, in the Medical and Surgical units, with hospitalized elderly. Collection carried out from February to June 2019. Applied instruments of sociodemographic data, assessment of functional capacity, in addition to physical examination of the skin. Results: Final sample of 32 elderly people, men (56.3\%), average age of $68.90( \pm 7.51)$ and mixed race $(59.4 \%)$. In assessing the capacity for self-care, $34.5 \%$ were totally dependent and $18.7 \%$ needed partial help. It was observed other types of lesions besides the skin tear, considering that they were bedridden elderly and with fragile skin. The prevalence of patients with skin lesions was $9.4 \%$. Conclusion: Carrying out a thorough physical examination of the skin in the elderly and assessing the risk for skin tears during the hospitalization period are fundamental actions to ensure safety in care, in addition to the inclusion of functional capacity assessment, since the degree of dependence can be a risk factor for the development of this type of injury.
\end{abstract}

Key words: Wounds and injuries, Elderly, Activities of daily living, Nursing care.

\section{RESUMEN}

Objetivo: Identificar la ocurrencia de lesiones cutáneas por desgarro cutáneo y verificar su relación con el estado funcional de ancianos hospitalizados. Métodos: Estudio descriptivo realizado en un hospital universitario de Brasilia, Distrito Federal, en las Unidades Médica y Quirúrgica, con ancianos hospitalizados. Recolección realizada de febrero a junio de 2019. Instrumentos aplicados de datos sociodemográficos,

1 Universidade de Brasília (UnB), Brasília - DF. *E-mail: andreamathes@unb.br

SUBMETIDO EM: 10/2020

ACEITO EM: 11/2020

PUBLICADO EM: 2/2021 
valoración de la capacidad funcional, además de exploración física de la piel. Resultados: Muestra final de 32 ancianos, hombres $(56,3 \%)$, edad media de $68,90( \pm 7,51)$ y mestizos $(59,4 \%)$. Al evaluar la capacidad de autocuidado, el $34,5 \%$ eran totalmente dependientes y el 18,7\% necesitaba ayuda parcial. Se observó otro tipo de lesiones además del desgarro cutáneo, considerando que eran ancianos postrados en cama y con piel frágil. La prevalencia de pacientes con lesiones cutáneas fue del 9,4\%. Conclusión: La realización de un examen físico minucioso de la piel en el anciano y la evaluación del riesgo de desgarros cutáneos durante el período de internación son acciones fundamentales para garantizar la seguridad en el cuidado, además de la inclusión de la evaluación de la capacidad funcional, ya que el grado de dependencia puede ser un factor de riesgo para el desarrollo de este tipo de lesiones.

Palabras clave: Heridas y traumatismos, Ancianos, Actividades cotidianas, Atención de enfermería.

\section{INTRODUÇÃo}

As lesões do tipo skin tears, nomenclatura mais reconhecida internacionalmente, trata-se de uma lesão de pele que tem causa traumática, com etiologia resultante pela associação da força de fricção e o cisalhamento, o que resultará na separação da epiderme da derme ou na segmentação de ambas as estruturas subjacentes. Pode ser mais comum em populações que possuem alterações relacionadas à fragilidade física, como ter a pele mais frágil, ser mais dependente para o autocuidado, possuir alterações nas condições de mobilidade e nutrição, como nos casos de pessoas idosas e de pessoas em cuidados de terminalidade. Os fatores intrínsecos para o desenvolvimento das skin tears incluem idade, raça, sexo, irradiação ultravioleta por exposição ao sol, desidratação, má nutrição, medicamentos, comprometimento cognitivo, mobilidade alterada e condições da pele, como púrpura senil (hematomas) (KOYANO Y, et al., 2017; STRAZZIERI-PULIDO KC, et al., 2015; AMARAL AFS, et al., 2012).

As taxas de prevalência das skin tears tem sido relatada entre 3 a 14\%, entre pessoas hospitalizadas ou em cuidados de longa duração. Os pacientes afetados desenvolvem complicações importantes, como infecções secundárias de feridas, que podem ser extremamente debilitantes, com consequente impacto negativo na qualidade de vida e aumento dos custos para o sistema de saúde por estadias prolongadas relacionadas à hospitalização (SERRA R, et al., 2018; KOYANO Y, et al., 2017; LEBLANC K, et al., 2013).

Nos idosos, ocorrem alterações fisiológicas e patológicas da pele: a pele mais velha apresenta menos elastina de colágeno e tecido adiposo, com diminuição da elasticidade da pele e redução do tecido subcutâneo o que ocasiona o aparecimento de rugas e dobras; há uma diminuição em número e atividade das glândulas sebácea e sudoríparas o que promove o ressecamento da pele, levando a xerose. A pele então se torna mais frágil e alterações arterioscleróticas nos pequenos e grandes vasos causam afinamento das paredes vasculares e consequente redução do suprimento sanguíneo para as extremidades e à microcirculação da pele. Pelo fato desta pele estar mais fragilizada as lesões por atrito podem ser mais comuns, que são aquelas provocadas por um trauma mecânico, também denominadas skin tears (MESAARANGO AC, et al., 2017; ARANHA JS, et al., 2020).

Alterações sensoriais (déficits cognitivos, auditivos e visuais) e comprometimento da mobilidade também têm sido relacionados às lágrimas da pele, mas não há estudos específicos sobre seus mecanismos fisiopatológicos que provavelmente estejam relacionados aos mesmos problemas das lesões por pressão. História de quedas, mobilidade prejudicada e consequente incapacidade de realizar atividades da vida diária e trauma mecânico têm sido frequentemente encontradas na história clínica de pacientes com skin tears (SERRA R, et al., 2018).

Fatores mecânicos é um problema real no aparecimento das skin tears, em muitas situações estas lesões podem ocorrer quando os profissionais de saúde lidam com os pacientes, durante uma movimentação por exemplo, ou quando removem fitas cirúrgicas, pois serem intervenções que se aplicam forças externas à superfície da pele, principalmente em pessoas idosas. De fato, os pacientes e suas famílias devem estar envolvidos no desenvolvimento e implementação de estratégias de prevenção, e a equipe de enfermagem e 
os cuidadores devem ser educados para garantir que estejam adotando técnicas corretas durante as práticas de cuidados com a pele, a fim de evitar lesões na pele, do tipo as skin tears (SERRA R, et al., 2018; ARANHA JS, et al., 2020).

Neste sentido, a presente pesquisa teve por objetivos identificar a ocorrência das lesões de pele do tipo skin tears e verificar sua relação com o estado de funcionalidade em idosos hospitalizados.

\section{MÉTODOS}

Trata-se de estudo descritivo com análise quantitativa. O estudo foi realizado em um hospital universitário na cidade de Brasília, Distrito Federal, nas unidades de internação da Clínica Médica e da Clínica Cirúrgica, sendo a população estudada a de idosos, convidados a participar de forma voluntária conforme admissão nas unidades.

O período da coleta de dados se deu entre os meses de fevereiro a junho de 2019. Como critérios de inclusão foram considerados os idosos, ou seja, pessoas com 60 anos ou mais, e que estivessem hospitalizados durante o período da coleta, sendo assim uma amostra de conveniência. A coleta foi realizada por meio da aplicação de instrumento elaborado pelos pesquisadores que constava de dados demográficos e pelo exame físico da pele para identificação das lesões de pele, do tipo skin tear, quando houvesse a presença da mesma.

Para identificação das lesões do tipo skin tears foram consideradas a classificação dada pelo instrumento Skin Tear Classification System (STAR), que inclui cinco categorias e subcategorias fundamentado na viabilidade e existência de fragmento ou retalho de pele, contendo um protocolo de tratamento da ferida, além do sistema de classificação e glossário. O STAR possui cinco fotografias, sendo cada uma delas relacionadas a uma descrição específica para a skin tear, a versão em português foi validada no ano de 2015, destinada a prática clínica para a identificação destas lesões (STRAZZIERI-PULIDO KC, et al., 2015).

A capacidade funcional para as atividades básicas de vida diária (ABVD) foi avaliada por meio da aplicação do instrumento proposto por Katz que avalia o nível de dependência da pessoa para desempenhar um conjunto de seis atividades diárias: tomar banho, vestir-se, higiene pessoal, transferência, continência e alimentação.

O instrumento possui três categorias de classificação para cada atividade, e, ainda em sua classificação são utilizadas 7 classes, distribuídas em letras que vão de A a G. Cada letra corresponde a um nível de funcionalidade sendo elas: independente $(A$ e $B)$, parcialmente dependente $(C, D$ e $E)$ e totalmente dependente ( $\mathrm{F}$ e G) (SIMÕES AL, et al., 2018; DUARTE YAO, et al., 2007).

A análise dos dados foi efetuada por meio de estatística descritiva para se obter a frequência e as relações das variáveis adstritas do estudo. A organização dos dados foi realizada por meio da categorização e transcrição das informações coletadas para planilha eletrônica Excel, da suíte Microsoft, onde também foram realizadas as análises.

O projeto de pesquisa foi submetido à apreciação do Comitê de Ética em Pesquisa da Faculdade de Ciências da Saúde da UnB, e obteve aprovação sob o número do Certificado de Apresentação para Apresentação Ética (CAAE) n 78558017.5.0000.0030.

\section{RESULTADOS}

Foram incluídos no estudo 32 idosos, a maioria eram homens $(56,3 \%)$, estavam na faixa etária entre os 60 aos 69 anos $(62,5 \%)$, com média de idade de $68,90( \pm 7,51)$, sendo um grupo de idosos jovens. Em relação à cor autodeclarada a mais prevalente foi a parda (59,4\%). Sobre a escolaridade dos idosos incluídos no estudo a maioria possuía de 1 a 4 anos $(34,4 \%)$ seguidos daqueles que possuíam de 5 até 8 anos $(25 \%)$. Quanto à renda familiar $78,1 \%$ recebia até um salário mínimo mensal. Em relação à capacidade de autocuidado para as atividades básicas de vida diária $46,8 \%$ eram totalmente independentes para todas as atividades (Tabela 1). 
Tabela 1 - Distribuição de dados sociodemográficos e capacidade funcional em idosos hospitalizados. Brasília, Distrito Federal, 2019 ( $n=32)$.

\begin{tabular}{|c|c|c|c|}
\hline Variáveis & $\mathbf{n}$ & $\%$ & Média ( $\pm \mathrm{DP})$ \\
\hline \multicolumn{4}{|l|}{ Sexo } \\
\hline Masculino & 18 & 56,3 & \\
\hline Feminino & 14 & 43,7 & \\
\hline \multicolumn{4}{|l|}{ Faixa Etária } \\
\hline 60 a 69 anos & 20 & 62,5 & \multirow{4}{*}{$68,90( \pm 7,51)$} \\
\hline 70 a 79 anos & 8 & 25,0 & \\
\hline 80 a 89 anos & 3 & 9,3 & \\
\hline 90 a 99 anos & 1 & 3,2 & \\
\hline \multicolumn{4}{|l|}{ Cor } \\
\hline Branca & 3 & 9,3 & \\
\hline Parda & 19 & 59,4 & \\
\hline Preta & 8 & 25,0 & \\
\hline Amarela / Indígena & 2 & 6,3 & \\
\hline \multicolumn{4}{|l|}{ Anos de Escolaridade } \\
\hline 1 até 4 anos de estudos & 11 & 34,4 & \multirow{5}{*}{$5,09( \pm 3,88)$} \\
\hline 5 até 8 anos de estudos & 8 & 25,0 & \\
\hline 9 até 11 anos de estudos & 2 & 6,2 & \\
\hline Sem instrução & 7 & 21,8 & \\
\hline Acima de 11 anos de estudos & 4 & 12,5 & \\
\hline \multicolumn{4}{|l|}{ Renda } \\
\hline Não possui renda & 4 & 12,5 & \\
\hline Até 1 salário mínimo & 25 & 78,1 & \\
\hline De 1 a 2 salários mínimos & 3 & 9,4 & \\
\hline \multicolumn{4}{|l|}{ Atividades Básicas de Vida Diária } \\
\hline Independente (A e B) & 15 & 46,8 & \\
\hline Parcialmente Dependente (C, D, E e F) & 6 & 18,7 & \\
\hline Dependente (G e H) & 11 & 34,5 & \\
\hline
\end{tabular}

Fonte: Galvão ACB, et al., 2020.

Pode-se notar através do estudo que a maior parte dos idosos entrevistados apresentava alguma lesão de pele. E durante o exame físico foi possível observar além da skin tears outros tipos de lesões, considerando que eram idosos acamados e com a pele fragilizada. Foi observado a lesão por pressão em diferentes estágios de evolução (estágio 1 e 2) e outros pacientes com diabetes mellitus, com lesões no pé. A prevalência de lesão por pressão foi de $40,6 \%$, enquanto a prevalência de pacientes com lesão por pé diabético era de $9,4 \%$. Já para as skin tears também foi uma prevalência de $9,4 \%$. Os que não apresentavam lesão de pele representaram $40,6 \%$ de todos os idosos avaliados (Tabela 2).

Tabela 2 - Distribuição de lesões de pele em idosos hospitalizados. Brasília, Distrito Federal, 2019 (n=32).

\begin{tabular}{lcc}
\hline Lesões de pele & $\mathbf{n}$ & $\%$ \\
\hline Lesão por pressão & 13 & 40,6 \\
Lesão por pé diabético & 3 & 9,4 \\
Skin Tears & 3 & 9,4 \\
Ausência de lesões de pele & 13 & 40,6
\end{tabular}

Fonte: Galvão ACB, et al., 2020.

Ao relacionar a capacidade funcional, que é a realização das atividades básicas de vida diária em relação à presença de skin tears, foi possível observar que todos os idosos que apresentaram a lesão ou eram dependentes $(18,18 \%)$ ou eram parcialmente dependentes $(16,7 \%)$ Apesar de ser uma amostra de idosos mais jovens, foi possível observar um elevado grau de dependência total ou parcial $(53,25)$ para as atividades de autocuidado e assim um risco aumentado para o desenvolvimento de lesões de pele, que resultam das forças de friç̧ão e cisalhamento na pele, principalmente entre idosos que precisam de auxílio para realizar mobilizações e transferências durante a hospitalização (Tabela 3). 
Tabela 3 - Relação entre a presença de skin tears e a capacidade funcional em idosos hospitalizados. Brasília, Distrito Federal, 2019 ( $n=32)$.

\begin{tabular}{lccc}
\hline \multicolumn{1}{c}{ Variáveis } & \multicolumn{3}{c}{ Presença de skin tears } \\
& $\begin{array}{c}\text { Sim } \\
\mathbf{n}(\%)\end{array}$ & $\begin{array}{c}\text { Não } \\
\mathbf{n}(\%)\end{array}$ & $\begin{array}{c}\text { Total } \\
\mathbf{n}(\%)\end{array}$ \\
\hline Capacidade Funcional & & & \\
\hline Dependente & $2(18,18)$ & $9(81,82)$ & $11(34,5)$ \\
Parcialmente Dependente & $1(16,7)$ & $5(83,3)$ & $6(18,7)$ \\
Independente & $0(0,0)$ & $15(100,0)$ & $15(46,8)$ \\
\hline
\end{tabular}

Fonte: Galvão ACB, et al., 2020.

\section{DISCUSSÃO}

Cuidados com as lesões da pele são uma realidade rotineira das atividades da enfermagem. Isso exige um constante acompanhamento das condições do paciente, devendo a equipe de enfermagem estar atenta às mudanças diárias e aos fatores de risco para o surgimento de uma lesão da pele, como é o caso da skin tears (TRISTAO FR, et al., 2020).

Dentre as lesões de pele, as lesões por fricção ou skin tears são um desafio para a enfermagem, infelizmente em muitas realidades são negligenciadas pelas equipes e pelas instituições hospitalares. Os idosos são os mais propensos às skin tears, principalmente devido a capacidade funcional reduzida associada às alterações histológicas da pele como redução da espessura da pele, da hidratação, da elasticidade e da resistência, alterações que se potencializam depois dos 75 anos (VIEIRA CPB, et al., 2020).

A prevalência de skin tears em idosos neste estudo foi de 9,4\%. Um estudo de revisão de literatura apontou que a prevalência de skin tears variou de 3,3\% a 22\% no ambiente hospitalar e 5,5\% a 19,5\% no ambiente domiciliar (STRAZZIERI-PULIDO KC, et al., 2015). Destacamos que as mesmas podem acabar gerando dor e podem se tornar crônicas e infectar, com isso, impactando diretamente na qualidade de vida. As skin tears tem ganhado cada vez mais espaço no debate em eventos sobre lesões de pele, que por muitos a consideram mais frequentes que as queimaduras e lesão por pressão.

Alguns pesquisadores afirmam que as skin tears possui uma prevalência maior que as a lesões por pressão, e que por serem entendidas como incidentes casuais inevitáveis ou por estarem associadas aos idosos que possuem uma pele mais frágil acabam sendo encaradas como algo irrelevante (ARANHA JS, et al., 2020; STRAZZIERI-PULIDO KC, et al., 2015; SANTOS EI, 2014). A subnotificação e negligencia em relação a skin tears acaba levando ao aumento da dor e sofrimento do paciente e prolongando o tempo de recuperação e de internação (ARANHA JS, et al., 2020; STRAZZIERI-PULIDO KC, et al., 2015; TIGGELEN HV, et al., 2020).

Ter outras alterações na pele como a presença de púrpura senil, equimoses e edema, presença de lesão por pressão, além de possuir comorbidades associadas como diabetes mellitus, pode haver a diminuição da percepção da dor e da sensibilidade tátil, pela presença da neuropatia diabética (POWELL RJ, et al., 2017). Situação observada entre os idosos da presente pesquisa no qual além da skin tears, $40 \%$ apresentava lesão por pressão em algum estágio, e lesão por pé diabético.

O idoso está mais exposto as skin tears do que qualquer outro grupo devido às alterações histológicas da pele e a diminuição da capacidade funcional (SERRA R, et al., 2015; CÉSAR CC, et al., 2015). A identificação dos pacientes em risco e a implementação de protocolos de prevenção tem se provado como capaz de prevenir ou minimizar os agravos pelas skin tears tendo como um elemento importante para o risco a avaliação do grau de dependência do idoso (ARANHA JS, et al., 2020; STRAZZIERI-PULIDO KC, et al., 2015; BARBOSA BR, et al., 2014).

Cabe ao enfermeiro, logo na admissão hospitalar realizar esse tipo de cuidado e avaliação a fim de reconhecer as alterações histológicas presentes na pele e o grau da redução da capacidade funcional do idoso, e assim elaborar o plano de cuidados, com vistas a proteção e segurança durante o período de hospitalização. 
As alterações histológicas da pele do idoso são causadas por fatores intrínsecos e extrínsecos. Nos fatores intrínsecos temos a diminuição das células de Langerhans e na densidade dos melanócitos; diminuição da junção dermo-epidérmica (sistema de ancoragem entre as camadas da derme e da epiderme) que faz com que não tenha uma boa resistência às forças mecânicas como as forças de fricção e cisalhamento. Alterações nas fibras de colágeno que leva a pele a perder sua força tênsil, resistência, elasticidade e extensibilidade.

Bem como modificações nas forças mecânicas tegumentares com redução da produção das glândulas sudoríparas e sebáceas o que torna a pele desidratada, ressecada, sem elasticidade, e diminuição da espessura da camada subcutânea, levando a diminuição da eficiência na absorção de impactos sobre a mesma. Quanto aos fatores extrínsecos temos o fotoenvelhecimento já que a exposição à radiação ultravioleta leva ao adelgaçamento e alterações na síntese de colágeno, colaborando com a fragilidade da pele e aumentando o risco de skin tears. Esses fatores diminuem a capacidade da pele a resistir a forças de tração, fricção e compressão e a forças traumáticas (STRAZZIERI-PULIDO KC, et al., 2015; MESA-ARANGO AC, et al., 2017; MENOITA E, et al., 2013).

$\mathrm{Na}$ avaliação da capacidade funcional dos idosos, 53,2\% possuíam algum tipo de dependência para pelo menos uma das atividades de vida diária (parcial ou total). A diminuição da capacidade funcional com consequente dependência na realização das atividades básicas de autocuidado está associada como fatores de risco para o desenvolvimento das skin tears em pessoas com a idade avançada associadas à presença da fragilidade, redução da mobilidade física e função cognitiva (STRAZZIERI-PULIDO KC, et al., 2015; MESA-ARANGO AC, et al., 2017).

Os idosos totalmente dependente acabam tendo maior necessidade de auxilio durante a realização de atividades do cotidiano, como banhar-se, vestir-se, transferir-se e posicionar-se na cama ou cadeira, atividades que podem promover aumento na tração da pele. Entre as áreas do corpo que estão mais expostas as skin tears estão os membros superiores e inferiores que são os mais mobilizados durante este tipo de atividade (STRAZZIERI-PULIDO KC, et al., 2015; TRISTAO FR, et al., 2020; VIEIRA CPB, et al., 2020). Entre os locais de maior ocorrência das skin tears estão o dorso das mãos, os braços, os cotovelos (nos membros superiores), região dorso glúteo e as pernas (nos membros inferiores), com formação de exsudato seroso e dor nas primeiras 24 horas (ARANHA JS, et al., 2020; SANTOS EI, 2014).

Apesar das lesões nos braços serem mais comuns, as lesões nas pernas podem evoluir para úlceras nas pernas, o que pode exigir um tratamento mais longo e caro. As causas típicas para o aparecimento das skin tears em idosos incluem uso de cadeiras de rodas, outros auxiliares de locomoção, bater em obstáculos, transferências e quedas. Existem diretrizes de melhores práticas para o tratamento e prevenção de infecções e úlceras. A prevenção inclui educação da equipe, avaliação regular, garantindo que a roupa não esfregue na pele, remover obstáculos e hidratar a pele do idoso diariamente, especialmente após o banho. Além disto, o uso de meias de proteção nas pernas se mostrou como uma estratégia já comprovada em estudos internacionais para prevenção das skin tears (POWELL RJ, et al., 2017; POWELL RJ, et al., 2015).

No que se refere ao cuidado da enfermagem, a avaliação da capacidade funcional do idoso tem o papel em identificar as condições de declínio físico, tendo como objetivo evitar lesões de pele e suas complicações. A identificação prévia de idosos com incapacidade funcional permite a melhoria da qualidade de vida, redução da morbidade e da mortalidade associadas aos cuidados específicos as demandas de um idoso com maior grau de dependência. Vale ressaltar que as lesões tissulares representam um grande problema de saúde pública, pois acarreta um aumento no tempo de internação, custo financeiro para o setor saúde, além de prolongar o tempo para a recuperação durante uma hospitalização (STRAZZIERI-PULIDO KC, et al., 2015; SANTOS EI, 2014; CAMACHO ACLF, et al., 2015; JOAQUIM FL, et al., 2016).

Ressaltamos que avaliação da capacidade funcional do idoso é importante em todos os aspectos do cuidado a ser prestado principalmente em unidades de internação hospitalar, sendo que a incapacidade para as atividades de autocuidado é um fator de risco para a mortalidade entre esta população (SIMÕES AL, et al., 2018). Destacamos que a hospitalização é um fator reconhecido como risco para o declínio funcional da pessoa idosa, potencializando o comprometimento funcional pré-existente (VIEIRA CPB, et al., 2020). 
Um elemento que é necessário também destacar é a variável escolaridade. A baixa escolaridade pode ser um fator de dificuldades na compreensão e assimilação dos cuidados relacionados a pele e lesões, tanto no ambiente de internação quanto no domicilio, interferindo assim na compreensão por parte de idosos e seus cuidadores sobre a doença e seus cuidados mais específicos (CAMACHO ACLF, et al., 2015). Fato que foi observado na presente pesquisa, sendo a maioria dos idosos incluídos com apenas 5,09 anos de estudo em média.

Assim é fundamental, que enfermeiros e a equipe de enfermagem, desenvolvam estratégias de ensinoaprendizagem voltadas para a promoção da saúde que possam abarcar as características de escolaridade, condições econômicas e sociais do público alvo que estão sob os seus cuidados, o que permitirá aos pacientes e seus familiares aprender a lidar com os fatores que possam interferir na prevenção e cicatrização de lesões de pele, como é o caso das skin tears (CAMACHO ACLF, et al., 2015).

A elevada prevalência das lesões do tipo skin tears no presente estudo, deve ser considerada pela equipe de enfermagem durante o planejamento do plano terapêutico ao idoso, incluindo o processo de enfermagem. Além disto, este plano de cuidados precisa ser compartilhado com toda equipe de saúde para que todos possam contribuir com o sucesso terapêutico, incluindo todos os profissionais que atendam este idoso bem como familiares e cuidadores (SANTOS EI, 2014).

A melhor prática de cuidados das skin tears inclui a identificação precoce e precisa, classificação, documentação e a aplicação de um protocolo de tratamento baseado em evidências. Para otimizar o manejo desde o estágio mais precoce possível de atendimento, recomenda-se a avaliação sistemática das lesões cutâneas usando uma ferramenta de classificação, a qual deve ser incluída na avaliação de admissão de idosos em unidades de internação hospitalar (TIGGELEN HV, et al., 2020, TRISTAO FR, et al., 2020).

\section{CONCLUSÃO}

A enfermagem tem um papel central na prevenção e cuidado com as skin tears junto a idosos mais dependentes. Para isso é fundamental que o enfermeiro tenha o conhecimento necessário para realizar medidas de prevenção e cuidados específicos com esse tipo de lesão de pele, por se tratar de uma lesão de alta prevalência entre idosos dependentes de auxílio e cuidados em suas atividades básicas de vida diária. A realização de um exame físico minucioso na pele do paciente idoso e o levantamento do risco para skin tears durante o período de hospitalização, são ações fundamentais para garantir a segurança no cuidado, além da inclusão da avaliação da capacidade funcional, já que que o grau de dependência pode ser um fator de risco que possa expor pessoas idosas ao desenvolvimento de skin tears.

\section{REFERÊNCIAS}

1. AMARAL AFS, et al. Prevalência de lesões por fricção em pacientes hospitalizados com câncer. Rev. esc. enferm. USP. 2012, 46(spe):44-50.

2. ARANHA JS, et al. Intervenções de enfermagem na prevenção de skin tears / Skin tears prevention nursing interventions. Braz J Dev. 2020;6(6):36849-60.

3. BARBOSA BR, et al. Avaliação da capacidade funcional dos idosos e fatores associados à incapacidade. Ciênc Saúde Coletiva.2014;19:3317-25.

4. CAMACHO ACLF, et al. Estudo comparativo sobre a capacidade funcional de pacientes adultos e idosos com úlceras venosas. Rev Pesqui Cuid É Fundam Online. 2015;7(1):1954-66.

5. CÉSAR CC, et al. Capacidade funcional de idosos: análise das questões de mobilidade, atividades básicas e instrumentais da vida diária via Teoria de Resposta ao Item. Cad Saúde Pública. 2015;31(5):931-45.

6. DUARTE YAO, et al. O Índex de Katz na avaliação da funcionalidade dos idosos. Rev. esc. enferm. USP. 2007; 41( 2 ): 317-325.

7. JOAQUIM FL, et al. Impacto da visita domiciliar na capacidade funcional de pacientes com úlceras venosas. Rev. Bras. Enferm. 2016, 69 (3): 468-477.

8. KOYANO Y, et al. Skin property can predict the development of skin tears among elderly patients: a prospective cohort study - PubMed. Int Wound J. 2017;14(4):691-7.

9. LEBLANC K, et al. Prevalence of skin tears in a long-term care facility. Wound Ostomy Continence Nurs. 2013;40(6):580-4.

10. MENOITA E, et al. A pele na pessoa idosa. Journal of Aging \& Inovation, 2013;2(1):18-33.

11. MESA-ARANGO AC, et al. Mecanismos de envejecimiento de la piel. latreia. 2017;30(2):160-70. 
12. POWELL RJ, et al. Pilot parallel randomised controlled trial of protective socks against usual care to reduce skin tears in high risk people: 'STOPCUTS'. Pilot Feasibility Stud. 2017;3:43.

13. POWELL RJ, et al. "Pilot randomised controlled trial of protective socks against usual care to reduce skin tears in high risk people "STOPCUTS": study protocol." Pilot and feasibility studies. 2015, 1:12.

14. SANTOS El. Cuidado e prevenção das skin tears por enfermeiros: revisão integrativa de literatura. Rev Gaúcha Enferm. 2014,35(2):142-9.

15. SERRA R, et al. Skin tears and risk factors assessment: a systematic review on evidence-based medicine. Int Wound J. 2018;15(1):38-42

16. SIMÕES AL, et al. Medição da autonomia em atividades da vida diária. Port J Public Health. 2018;36(1):9-15.

17. STRAZZIERI-PULIDO KC, et al. Skin tear prevalence and associated factors: a systematic review. Rev Esc Enferm USP. 2015;49(4):0674-80.

18. STRAZZIERI-PULIDO KC, et al. Adaptação cultural, validade de conteúdo e confiabilidade interobservadores do "STAR Skin Tear Classification System". Rev. lat.-am. enferm.2015;23(1):155-61.

19. TIGGELEN HV, et al. Standardizing the classification of skin tears: validity and reliability testing of the International Skin Tear Advisory Panel Classification System in 44 countries. Br J Dermatol. 2020;183(1):146-154.

20. TRISTÃO FR, et al. Práticas de cuidados do enfermeiro na atenção primária à saúde: gestão do cuidado da pele do idoso. Cogitare Enferm. 2020;25(0).

21. VIEIRA CPB, et al. Prevalência de lesões por fricção e fatores associados em idosos em terapia intensiva. Texto \& Contexto Enferm. 2020;29,e20180515. 